Available online on 15.12.2020 at http://jddtonline.info
OJDDT* $5 \%$

Open $\odot$ Access

Review Article

\title{
An Insight to Nanostructured Lipid Carrier System
}

\author{
G. Rajalakshmi*1, C.K. Dhanapal2, R. Sundhararajan ${ }^{3}$ \\ ${ }^{1}$ Associate Professor in Mohamed Sathak A.J College of Pharmacy, Sholinganallur, Chennai 119, India \\ 2 Professor, Department of Pharmacy, Annamalai University, Chidambaram, Annamalai Nagar 002, India \\ ${ }^{3}$ Principal, Mohamed Sathak A.J College of Pharmacy, Shollinganallur, Chennai 119, India
}

\begin{abstract}
In pharmaceutical field, many drugs are being invented to combat the existing new diseases. The winds of change in the drug scenario are blowing forcefully worldwide. The emergence of new technologies provides an unique opportunities to exploit novel approaches in drug delivery. A shift from conventional drug delivery to novel drug delivery is noticed as shift from conventional drug delivery suffers from various drawbacks, But these new mighty compounds in drug therapy solely are not sufficient to meet the today's need. There is an urgent need for the smart technology, as the drugs available suffer from serious problems like poor solubility and poor bioavailability. Most of the drugs that are available therapeutically comes under BCS class II ie, poorly soluble and high lipophilc. To assure progress in drug therapy, the development of new drugs merely is not sufficient. Issues arising in delivery of new drugs should also be addressed. Thus there is an emergent need to improve the bioavailability of these drugs, the only remedy or boon for such drugs is the discovery of some smart technologies which can improve the bioavailability of these drugs. Nano drug delivery systems are the one such universal approach which fulfills the lacuna, which exists in conventional drug delivery systems. These Nano drug delivery systems, improves the pharmacokinetic profiles of many drugs. In $1980 \mathrm{~K}$. Eric Drexler developed and popularized the concept of nanotechnology. In this review a deep insight on Nano structured lipid carriers is discussed elaborating its birth, significant qualities compared to other colloidal systems, its structure, characteristics, preparation and application are spotted.
\end{abstract}

Key words: BCS class, Lipophilic, Nano technology, Nano structured lipid carrier.

Article Info: Received 11 Oct 2020; $\quad$ Review Completed 16 Nov 2020; $\quad$ Accepted 23 Nov 2020; Available online 15 Dec 2020



Cite this article as:

Rajalakshmi G, Dhanapal CK, Sundhararajan R, An Insight to Nanostructured Lipid Carrier System, Journal of Drug Delivery and Therapeutics. 2020; 10(6-s):173-182 http://dx.doi.org/10.22270/jddt.v10i6-s.4589

*Address for Correspondence:

G. Rajalakshmi, Associate Professor in Mohamed Sathak A.J College of Pharmacy, Sholinganallur, Chennai 119, India

\section{INTRODUCTION:}

A pharmaceutical carrier scheme is needy with ideal characteristics like efficient drug loading capacity, free from toxicity, controlled release characteristics, drug targeting and good stability. Colloidal carriers like emulsions, liposomes, micro particles and niosomes suffered from certain flaws like stability issues, lack of production techniques, phagocytosis by reticulo endothelial system 1 . Nano carriers refer to materials prepared by the dissolution or dispersion of drugs with a variety of nanoparticles, which may be classified as either nanospheres or nanocapsules. The materials for the preparation of nanoparticles may be divided into polymers and lipid materials. The former is referred to as PNP, which includes polymer nanocapsules and nanospheres as well as polymeric micelles. The latter is called the lipid nanoparticle, and includes Nanoliposomes solid lipid nanoparticles and Nano structured Lipid carriers.

\section{BIRTH OF NLC}

Nano drug delivery systems may be of nanoparticles, nano carriers, nano emulsion or suspensions, fullerenes, nanotubes, quantum dots, nanopores, dendrimers, liposomes, magnetic nanoprobes and radio controlled nanoparticles.

Colloidal particles ranging in size between 10 and $1000 \mathrm{~nm}$ are known as nanoparticles. Initially synthetic/natural polymers were ideally used to manufacture .It suited to optimize drug delivery and reduce toxicity. Speiser and coworkers in the mid-seventies introduces polymeric nanoparticles. This developed as a variable substitute to liposomes as drug carriers after few years. Any nano drug delivery's success is by its ability to penetrate through several anatomical barriers, sustained release of their contents and their stability in the nanometer size. In spite of overwhelming advantages of polymeric nano particles, not CODEN (USA): JDDTAO 
many products made it to market because of certain stumbling blocks like presence of solvent residues left over from production, the cytotoxicity of the polymers, and the lack of low-cost, qualified large scale production units yielding a product of a quality acceptable by the regulatory authorities, the scarcity of safe polymers have limited the wide spread application of nanoparticles to clinical medicine $^{2}$

To overcome these limitations of polymeric nanoparticles, lipids have been put forward as an alternative carrier, particularly for lipophilic pharmaceuticals. ${ }^{3}$ These lipid nanoparticles are known as solid lipid nanoparticles (SLNs) developed in 1991. In 1999, the complete patent rights for production of SLN by high pressure homogenization have been acquired by SkyePharma4. Great progress was made in treating different diseases using SLN .SLNs are colloidal carriers developed in the last decade as an alternative system to the existing traditional carriers (emulsions, liposomes and polymeric nanoparticles). They are a new generation of submicron-sized lipid emulsions where the liquid lipid (oil) has been substituted by a solid lipid. SLN has special qualities such as small size, large surface area, high drug loading and the interaction of phases at the interfaces, and are attractive for their potential to improve performance of pharmaceuticals, neutraceuticals and other materials.

SLN are like nanoemulsions differing only in the nature of lipids .By replacing liquid lipid with solid lipid, the formulation achieved greater potential. This benefited with enoromous combined advantages of polymeric nanoparticles, fat emulsions, liposomes while avoiding their disadvantages. Being solid at room temperature, ease of dosing and handling, high meltingpoint waxes like glycerides or waxes form major constituent. 5-7 .Being an attractive colloidal carrier it overcome number of hurdles associated with conventional colloidal carriers.

\section{ADVANTAGES OF SLN:}

1. The size of sln is very significant, being in Nano size of $120-200 \mathrm{~nm}$, they bypass Reticulo endothelial system thus bypass liver and spleen. ${ }^{8}$

2. Analogy with colloidal carriers' solid lipid nanoparticles stay stable for even 3 years. ${ }^{9-10}$

3. Controlled release of drug can be accomplished for several weeks. Further by attaching or coating with ligands, drug targeting is also guarantied.11-15

4. Less toxic when compared with polymeric particles due to embodiment of physiological biocompatible lipids. ${ }^{16-}$ 18

5. Feasibility of incorporating both hydrophobic and hydrophilic drugs. 18

6. Due to immobilization of drug molecules by solid lipids the labile and sensitive drugs can be protected from chemical, photochemical or oxidative degradation. ${ }^{18}$

7. Excellent reproducibility with a cost effective highpressure homogenization method as the preparation procedure ${ }^{19}$

8. Improvement and reduction in the variability of GI absorption of poorly water-soluble, lipophilic drugs. ${ }^{20}$

9. Progress of sln is widespread. It find its applications in oral, parenteral, topical, peroral, transdermal, gene carrier therapy, cosmeceuticals,anti tubercular therapy, cancer therapy, proved as efficient in delivering antiretroviral drugs to brain,as apotential and new adjuvant for vaccines. ${ }^{21,22}$.

10. Ease of manufacturing and regulatory approval, low cost. $^{23}$

The trade name 'SLNTM' has been registered world-wide in the pharmaceutical area, as has 'LipopearlsTM' for cosmetics. These SLN were attracting number of formulators worldwide till they found out certain shortcomings or flaws in it.

\section{Drawbacks of SLN:24}

1. Limited drug loading capacity

2. Gellation tendency

3. Drug leakage during storage.

4. High water content of sln dispersion

5. Reduced particle concentration

6. Polymorphic transition.

7. Low incorporation due to crystalline structure of solid lipids.

\section{Particle growing}

To overwhelm the hurdles, at the turn of millennium, nanostructured lipid carriers or NLC, were developed to resolve, in some cases, the problems raised by SLNs. NLCs are produced by controlling the mixing of solid lipids with liquid oil, leading to special nanostructures in the matrix. The matrix of NLCs is composed of mixture of spatially different lipid molecules, normally mixture of solid and liquid lipid, which makes more imperfection in the matrix to accommodate more drug molecules than SLN.

NLC's are made up of a binary mixture of solid-lipid and a liquidlipid (oil) as a hybrid carrier having an average size of $10-500 \mathrm{~nm}$. The mixture NLC's is consist of long chain of liquid and lipid (oil) of ratio 99.9: 0.1 and having a short chain of solid and lipid having a ratio of 70:30 25-27.

These NLC appeared in late 1990's. This involves modification of SLNs by mixing solid and lipid nanoparticles followed by heating and cooling to form a stable system.

\section{Qualities of NLC:}

1. Despite of containing liquid lipid NLC behave as solids at room/ body temperatures. This is achieved by controlling the content of liquid lipid.

2. NLC strongly immobilize the drugs and prevent it from coalescing.

3. Particle size of NLC range from 10-1000nm.

4. The presence of liquid lipids with different fatty acid Cchains produces NLC with less organized crystalline structure and therefore provides better loading capacity for drug accommodation ${ }^{24}$

5. By high-pressure homogenization and the process can be modified to yield lipid particle dispersions with solid contents from $30-80 \%$.

6. Liquid carrier materials in NLC have a higher solvent capacity. This increased solvent capacity is due to additional active incorporation space created through the generation of crystal imperfections due to the presence of liquid lipid, in the highly ordered crystal structure of the solid lipid material 28 
7. A biphasic drug-release pattern is observed in NLC, which results from a rapid release of active from the liquid phase of the NLC, with a comparatively slower release of the API from the solid lipid portion of the NLC. This release pattern can be modulated by adjusting the proportions of the liquid and solid lipid carriers within the NLC. 29

8. The reason for good drug loading is, increased solubility of drug in liquid lipid than in solid lipid.

9. It has stable physical and chemical properties.

10. There is new and modified type of NLC's are available which is having a meticulous nanostructure. These meticulous nanostructures are responsible and also help to improve the stability of the formulations as well as increase the bioavailability, drug loading. ${ }^{30}$.

\section{ADVANTAGES OF NLC ${ }^{31-35}$}

1. A disordered crystal structure which prevents drug leakage.

2. A high drug pay load due to its microstructure.

3. The carrier material used is biodegradable and low in vivo toxicity.

4. The surface of NLC can be modified with surfactants for prolong release pattern

5. NLC's exhibits organizational targeting pattern

6. Better physical stability

7. Ease of preparation and scale-up,

8. Increased dispersability in an aqueous medium

9. High entrapment of lipophilic drugs and hydrophilic drugs

10. Controlled particle size

11. Furthermore, lipid nanoparticles are able to enhance the chemical stability of compounds sensitive to light, oxidation, and hydrolysis 32

12. BCS class II/IV drugs bioavailability is significantly increased by using this novel lipid transport system.

13. Extended release of the drug,

14. Small size of the lipid particles ensures close contact to the stratum corneum thus enhancing drug penetration into the mucosa or skin.

15. Nlc has slower polymorphic transition and low crystallinity index, membrane permeability and drug release, creaming, gelling, and particle aggregation during storage compared with $\sin { }^{33}$

16. Improve benefit/risk ratio, easy approval by regulatory agencies, easier to validate.

17. In case of topical preparations, give local action when applied. It also acts as local reservoir, controlled release of drug, as penetration enhancer. Due to small size increase penetration into gaps between stratum corneum cells. ${ }^{34}$

18. Studies have proven that this new Nano platform improved oral bioavailability drugs promoting their intestinal absorption. 35
19. This nano system has shed light of hope to treat many chronic diseases by modulating drug efficacy and sustained release of drug.

20. These lipid-drug conjugate (LDC) nanoparticles providing high loading capacities for hydrophilic drugs. Many researchers have proved these nlc are particularly useful for targeting water-soluble drugs to the brain.

\section{Limitations of NLC 36-37}

NLC being so successful in targeted drug delivery compared to SLN and other colloidal drug delivery systems, it also suffers from certain drawbacks like:

1. Cytotoxic effects related to the nature of matrix and concentration.

2. Certain surfactants used in formulation may have irritating and sensitizing action.

3. Still efficient methods are not there in NLC for delivering Protein, peptide and gene delivery.

4. NLC is still nascent, there is lack of sufficient clinical and preclinical trials in many areas particularly bone repair.

\section{MICRO STRUCTURE OF NLC: ${ }^{38-41}$}

In comparison with SLN, which is composed of perfect brick wall by using highly purified lipids the micro structure of NLC (shown in figure 1) is different disorgsnised structure. However, a high degree of spatial organization may lead to low drug loading. A more disorganized structure may be more advantageous. This microstructure disorganization leads to the presence of voids which can accommodate higher amount of drugs/bio active molecules. Such microstructural disorganization can be seen in Nano structured lipid carrier system (NLC) which is achieved by mixing solid lipid with liquid lipids or by using non purified raw materials instead of using highly purified lipids with relatively similar molecules.

Three types of NLC have been described: (I) imperfect type, (II) amorphous type and (III) multiple type

\section{I) Imperfect type NLC (imperfectly structured solid matrix):}

It is created when different types of lipid molecules are used to build the core of the particle. It is crystalline but presence of voids help to accommodate bioactive molecules or drugs.Mixing small amounts of chemically very different liquid lipids (oils) with solid lipids in order to achieve highest incompatibility leads the highest drug payload.

\section{(II) Amorphous type (structureless solid amorphous} matrix):

These are formed when particles are cooled they are not crystallize remaining in amorphous state. Therefore drug expulsion is minimized, as the transformation from polymorphic form to another does not occur.This kind of NLC can be achieved by mixing solid lipids with special lipids, e.g. hydroxyl octacosanyl hydroxystearate, isopropylmyristate or medium chain triglycerides such as Miglyol 812. 38

(III) Multiple types (multiple oil in fat in water $(0 / F / W)$ carrier):

This resembles multiple emulsion type in which solid lipid core contains liquid oil compartments. This type is observed when liquid lipid is more than solid lipid. At high temperature complete miscibility occurs but during cooling 
process precipitation occurs in the form of tiny droplets due to exceeding solubility of oil in solid lipid compound. Solubility of many drugs in a liquid lipid is higher than in a solid lipid. It has been reported that in case of lipids which lack appropriate drug solubilities, addition of a higher amount of liquid lipid to the lipophilic phase combined the advantages of the solid matrix which prevented drug leakage, and of the liquid regions (oily nanocompartments) which showed comparatively high solubility for lipophilic drugs $39-41$



a. Imperfect type

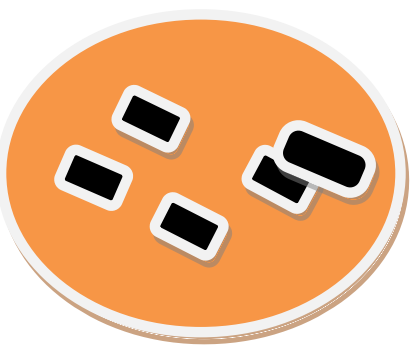

b. Amorphous type

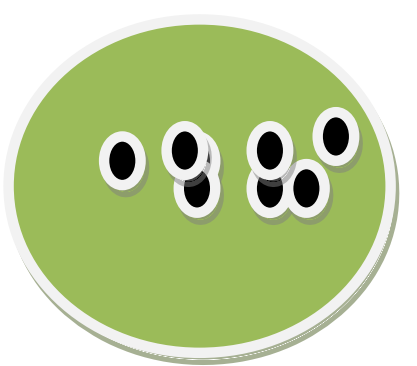

c. Multiple type

Figure 1 Different types of NLC

\section{Comparison between SLN and NLC ${ }^{42-44}$}

Table 1: Comparison between SLN and NLC

\begin{tabular}{|c|c|}
\hline SLN & NLC \\
\hline $\begin{array}{l}\text { Composed of solid lipid dispersed in aqueous medium, } \\
\text { stabilized by suractant }\end{array}$ & Composed of blend solid lipid and Liquid lipid (oils) \\
\hline $\begin{array}{l}\text { Ratio of solid lipid } 1-30 \% \mathrm{w} / \mathrm{w} ; \\
\text { Surfactant } 0.5-5 \% \mathrm{w} / \mathrm{w}\end{array}$ & Weight ratio of solid lipid and Liquid lipid 70:30 upto 90:10 \\
\hline Micro structure is highly ordered lipid matrix. & Microstructure is less ordered lipid matrix \\
\hline $\begin{array}{l}\text { Types of SLN } \\
\text { a. Type I or homogeneous matrix model, } \\
\text { b. Type II or drug-enriched shell model and } \\
\text { c. Type III or drug-enriched core model. }\end{array}$ & $\begin{array}{l}\text { Types of NLC } \\
\text { Imperfect type } \\
\text { Amorphous type } \\
\text { Multiple type }\end{array}$ \\
\hline $\begin{array}{l}\text { Structure of } \\
\text { SLN }\end{array}$ & $\begin{array}{l}\text { Structure of } \\
\text { NLC }\end{array}$ \\
\hline $\begin{array}{l}\text { Drug loading is less due to its high degree of order, the } \\
\text { number of imperfections in the crystal lattice is reduced } \\
\text { leading to drug expulsion }\end{array}$ & $\begin{array}{l}\text { Drug loading is high as lipid matrix is less ordered. Having } \\
\text { disordered structure provides higher space for drug loading } \\
\text { Also due to high solubility of drug in liquid lipids. }\end{array}$ \\
\hline $\begin{array}{l}\text { Drug expulsion due to polymeric transition occur during } \\
\text { storage }\end{array}$ & No drug expulsion \\
\hline High water content (70-90.9\%) & Less water content \\
\hline During storage loss of drug due to lipid crystallization & No loss of drug \\
\hline$\beta$ modification of lipid to metastable polymorph occur & No modification \\
\hline $\begin{array}{l}\text { Slower release of drug as mobility of drug in crystalline form } \\
\text { is less. }\end{array}$ & $\begin{array}{l}\text { Faster release of drug as mobility of drug in crystalline form is } \\
\text { less. }\end{array}$ \\
\hline
\end{tabular}




\section{INFLUENCE OF EXCIPIENTS IN NLC:}

The essential ingredients for the formulation of NLC are solid lipid, Liqid Lipid, Water and Emulsifier.

\section{Lipids:}

Lipids form the major constituents of NLC.

a. Soild lipid: They should be biodegradable, biocompatible, chemically stable without any toxic effects. The solid lipids used are solid at room temperature but melt at temperatures above $80^{\circ} \mathrm{C}$. The lipids used are given in Table no: The selection of lipids for formulating is done by testing the solubility of drug in lipid. Since it has direct impact on drug entrapment and loading efficiency. ${ }^{42}$ Moreover the stability of drug and lipid is also a key feature. Compatibility studies must be done between drug and lipid. There should not be any phase separation till 24 hours of mixing drug and lipid for acquiring stable NLC. 43

b. Liquid lipid: The Liquid lipids used are either digestible oils from natural sources or oily components.These should be biocompatible, cost effective, non irritating. All the lipids used should be approved by regulatory agencies as GRAS (generally recognized as safe). The solid lipid to liquid lipid typical weight ratio as per literature ranges from 70:30 to 90:10. A melting point depression occurs when liquid lipid mixes with and solid lipids compared to the pure solid lipid. A decrease in particle size was observed with increment in liquid lipid due to decrease in viscosity and high molecular mobility. The resulting structure remains solid at room temperature with API-loaded liquid pocket. ${ }^{44-46}$

Quantity of Liquid lipid play a significant role in tailoring particle size and release rate of NLC since it may lead to reduction in viscosity and surface tension thereby producing NLC with smaller size, high surface area and promoting high percent cumulative drug release ${ }^{47}$.
The liquid lipids used most commonly are listed in Table 2. Moreover vegetable oils, oils from plant sources, fish oils are gaining interest recently.

In short the role of lipids is salient frame work in NLC. With increase in lipid matrix, the particle size, entrapment efficiency are enhanced it may cause increase in viscosity, leading to reduction in drug escaping tendency and higher entrapment of drug. Thus selection of lipid contents is very pivotal in developing NLC with desired characteristics.

\section{Emulsifiers:}

In order to stabilize the system emulsifiers are used. Either lipophilic or hydrophilic emulsifiers are used. Combination of both can be used to prevent particle aggregation efficiently. They coat the surface of NLC there by providing long term stability. Emulsifiers are the fundamentals because they help in dispersing one immiscible phase with other. They tend to reduce the interfacial tension between two phases and increase the surface area of liquid droplets thereby yielding small particles. 48 The quantity of emulsifier added has influence on release kinetic profiles and entrapment efficiency. Reduction in interfacial tension by emulsifier occurs only up to a specified concentration after than increase in amount of emulsifier added leads to agglomeration may occur due to excess coating of particles and decrease in zeta potential. ${ }^{49-51}$ Emulsifiers with HLB value $\geq 18$ hydrophilic surfactants yields faster release pattern. A blend of surfactant is also used to enhance stability and viscosity of NLC. 52 In a nut shell selection of emulsifier, its concentration is significant in fabrication of NLC with effective drug delivery, reduced particles size, narrow size distribution assuring predictable drug release.

PEG is added in NLC to prevent uptake by reticuloendothelial system and to prolong the circulation time of drugs.

Table 2 Excipients used in NLC

\begin{tabular}{|c|c|}
\hline Excipients & Examples \\
\hline Solid lipid & 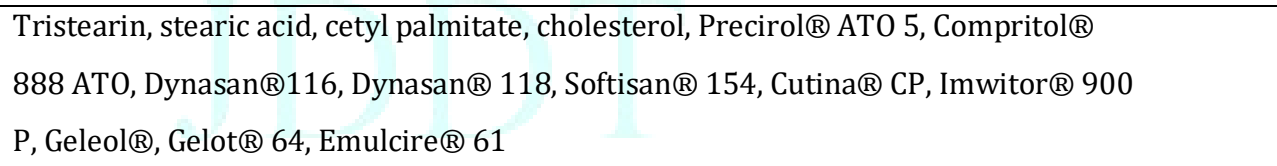 \\
\hline Liquid lipid & $\begin{array}{l}\text { Medium chain triglycerides, paraffin oil, 2-octyl dodecanol, oleic acid, squalene, } \\
\text { isopropyl myristate,vitamin E, Miglyol@ 812, Transcutol@ HP, Labrafil Lipofile }{ }^{\circledR} \\
\text { WL 1349, Labrafac® PG, Lauroglycol@ FCC,Capryol@ } 90\end{array}$ \\
\hline Lipophilic Emulsifier & Myverol ${ }^{\circledR}$ 18-04K, Span 20, Span 40, Span 60, \\
\hline Hydrophilic Emulsifier & 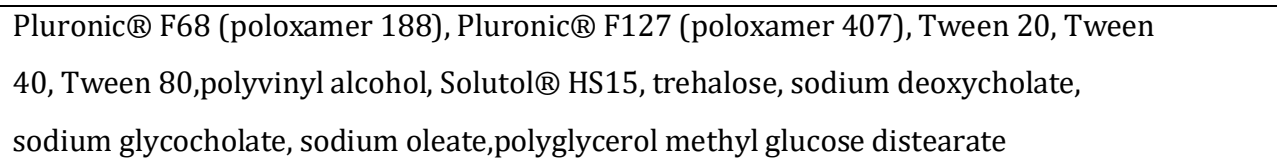 \\
\hline $\begin{array}{l}\text { Amphiphillic } \\
\text { Emulsifiers }\end{array}$ & Egg lecithin, soya lecithin, phosphatidylcholines, phosphatidylethanolamines, Gelucire ${ }^{\circledR}$ 50/13 \\
\hline
\end{tabular}

\section{MECHANISM OF DRUG RELEASE FROM NLC:}

Partition coefficient of drug, particle size, dispersion of drug in lipid matrix influences the drug release. The drug release behavior from NLCs is dependent upon the production temperature, emulsifier composition, and oil percentage incorporated in the lipid matrix. Drug release is inversely proportional to the partition coefficient of the drug. Decrease in particle size to nanometer scale causes increase in surface area, thus higher drug release. Drug should be dispersed equally in any lipid matrix for prolonged release of drug. 
Drug release from lipid particles occurs by diffusion and simultaneously by lipid particle degradation in the body. Apart diffusion and degradation, in some cases, it might be desirable to have a controlled fast release .The release can be triggered by an impulse also. Since NLCs are in highly unordered lipid structures, to initiate a burst drug release, there is a need to convert highly unordered lipid structure to more ordered structure. This can be accomplished by trigger impulse. By converting into a more ordered structure with the help of triggering impulse, a burst drug release occurs. ${ }^{53}$

This impulse can be of any thing such as increase in temperature, water evaporation in case of topical preparations. The drug amount in the outer shell of the nanoparticles and on the particulate surface is released in a burst manner, while the drug incorporated into the particulate core is released in a prolonged way. Sustained release of the drugs can be explained considering both drug partitioning between the lipid matrix and water, as well as the barrier function of the interfacial membrane. The dialysis method and the utilization of the Franz cell are the modes for measuring in vitro drug release from nanoparticles. The interpretation of in vitro drug release profiles should consider the specific environment in the in vivo status. Enzymatic degradation of lipid nanoparticles may be influenced to a relevant extent by the composition of the particles. ${ }^{54-55}$.



Figure 2: Modulation of drug release.

\section{A. SLN - Drug release as aggregates}

\section{DRUG DELIVERY 56-63}

The lymphatic system is part of the circulatory system, which is composed of an intricate network of conduits that carry lymph. The primary function of the system is to carry interstitial fluid accumulation back to the circulatory system and to transport immune cells to the lymph nodes. Drug incorporated in NLCs reach the systemic blood circulation through the intestinal lymphatic system, as opposed to entering the systemic blood circulation through the portal blood. By this two major benefits are reduction in first-pass metabolism and an increased overall bioavailability for the active drug. In addition to intestinal lymphatic uptake, NLCs can be delivered by means of other lymphatic pathways, including subcutaneous and pulmonary lymphatic uptake. Subcutaneous and pulmonary delivery of NLC have enormous advantages such as active accumulation at the site of administration, avoidance of first pass metabolism, prolonged release, low clearance, reduced dosing frequency, less systemic toxicity etc.,

Darunavir is effective against wild type and PI-resistant HIV, and has a very low oral bioavailability of $37 \%$. It is formulated with ritonavir to increase its bioavailability to $82 \%$. This low bioavailability is common for oral antiretrovirals, which typically have low-aqueous solubility, high CYP-mediated metabolism, and are often a substrate for Pglycoprotein efflux.64

In case of Topical delivery Nanoparticles with size more than $100 \mathrm{~nm}$ are difficult to penetrate into stratum cornem but uptake of these particles can occur. As the outer layer of skin is rich in lipids, exchange of lipids between stratum corneum and nano carriers may occur. The presence of follicles along

\section{B. NLC - Drug release in disperse manner}

with sebaceous glands promotes an environment rich in lipids. This helps in trapping lipid nano particles. Moreover sebum is a mixture of triglycerides, squalene and waxes. Some triglycerides present in NLC may accelerate entrance into follicles or sebaceous glands.

In case of oral delivery, drug loaded NLC pass through digestive system and lipid digestion occurs gradually. Initially triglycerides are broken into monoglycerides and free fatty acids by enzymatic action. The released bioactives are transported either actively or passively through enterocytes or they may enter lacteals through chylomicronmediated pathway. In case of chylomicrons pathway micelles are formed with amphipathic bile salts surrounding drug and monoglycerides. These micelles reach brush border of the intestine and can be absorbed. In the

Monoglycerides,fatty acids and bioactives diffuse out from micelles in the enterocytes leaving micelles behind and further reforming triglycerides inside intestinal cells. These lipid constituents in aggregation with cholesterol and phospholipids form chylomicrons encapsulating bioactives inside them, which finally undergo exocytosis.

Large size of chylomicrons (approximately $80 \mathrm{~nm}$ ) prevents their entry through blood capillaries and thus they enter via lacteals circumventing first pass metabolism of the bioactives associated with them. Particle size of NLCs also plays significant role in intestinal transport. The size below $300 \mathrm{~nm}$ is desirable for their transport across the intestine and should be suitably controlled. 


\section{Discrete techniques of NLC for its improved bioavailability:}

\section{Increased Permeability:64-65}

The surfactant employed in the preparation of NLC alters the permeability by various mechanisms. It inhibits $\mathrm{P}$-gp efflux transport and may increase intestinal permeability. Polaxmer used deforms the cell membrane and open epithelial cell junction and promote paracellular transport.

2. Inhibits Drug degradation:66

NLC offer protection to drugs from chemical and enzymatic degradation dealing in harsh GI environment.

3. Mixed Micelle formation
The lipids used in NLC are similar to dietary lipids and thus include in bile secretion in small intestine. The lipids which undergo degradation upon action of enzymes to form lipid digestion products and are mixed with bile to form micelle. Thus facilitating ease transportation. ${ }^{67}$

4. Muco adhesion characteristics: 68

Increased adhesion of carrier to mucus causes increased retention of drug release.

\section{Direct uptake:69-70.}

It is by intestinal lymphatic transport. NLC may stimulate chylomicron formation because of lipophilic transport this follows transcellular route of absorption. High lipophilicity promotes lymphatic intestinal absorption thereby avoiding first pass metabolism.

\section{PARAMETERS THAT INFLUENCE THE RELEASE PROFILES 27}
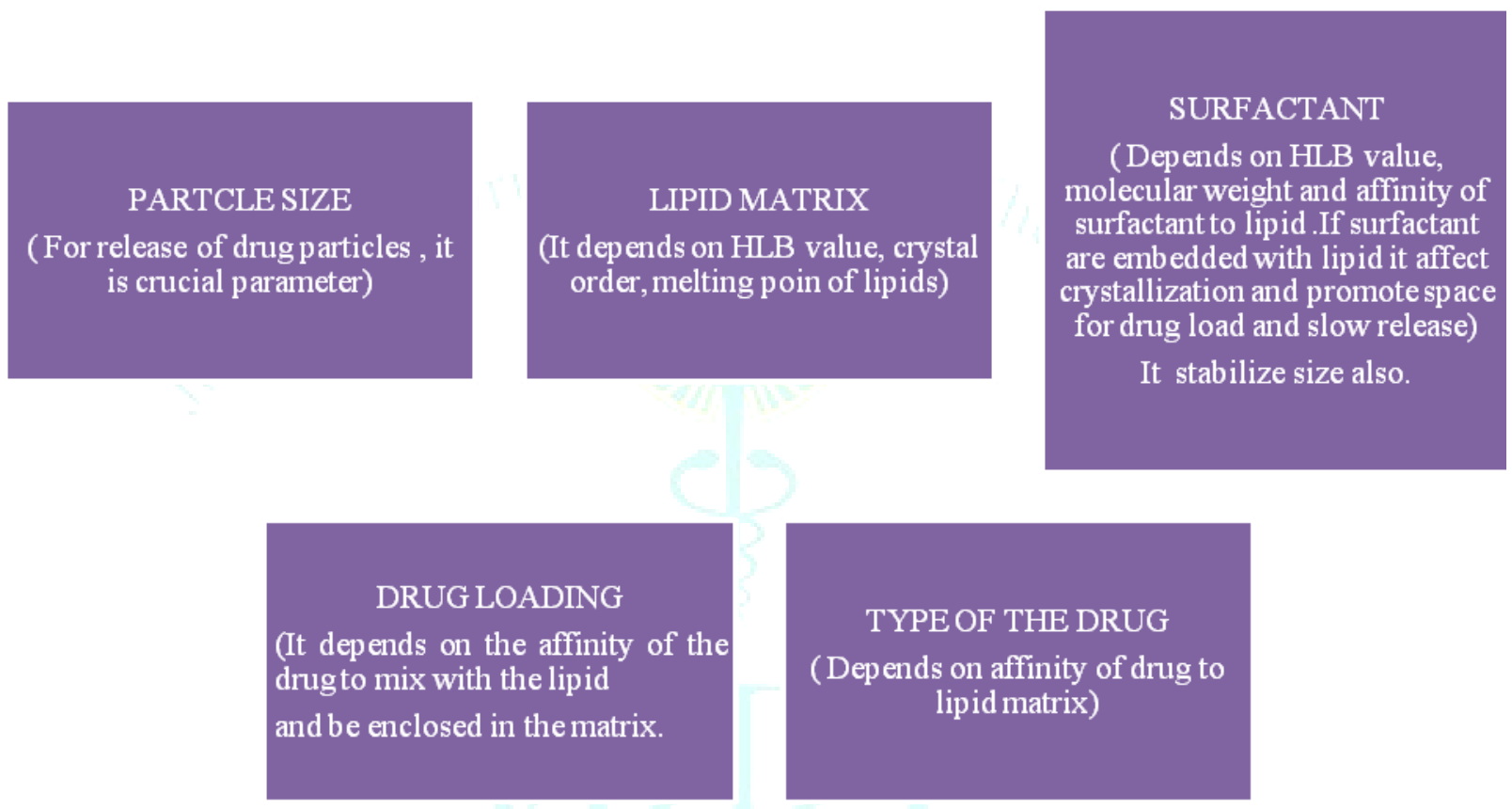

\section{PREPARATION 27,71}

There many methods for the preparation of lipid nanoparticulate drug delivery systems. The method used is dictated by the type of drug especially its solubility and stability, the lipid matrix, route of administration, etc.

1. Homogenization (Hot/cold)

2. Solvent Evaporation

3. Solvent emulsification and diffusion

4. Ultra sonication

5. Solvent injection

6. Phase inversion

7. Double emulsification

8. Melt dispersion

9. Supercritical fluid.

\section{Film ultra sound dispersion method}

\section{CHARACTERIZATION OF NLC: 72}

The formulated NLC's are characterized for physicochemical characterization as it is very essential to judge the feasibility of NLC's as ideal drug delivery system. The frequently used parameters are:

1. Particle size analysis: By Photon correlation spectroscopy and laser diffraction methods. The type and ratio of lipids have direct influence on particle size. The usage of emulsifier causes complete emulsification and thus reduces the particle size.

2. Zeta potential: Important parameter as it determines the aggregation and dispersion process affecting the stability. Presence of electric charge on surface prevents aggregation. For brain delivery positive charges are essential for efficient entry into blood brain barrier and to attach to anionic sites present in paracellular area over 
there. In certain cases negative charge NLC is essential for stabilization.

3. Morphology of particles: Particle size radius, size distribution, size and morphology of particles can be analysed by Scanning Electron Microscopy (SEM) and Transmission electron microscopy. Atomic force microscopy (AFM) is also used for measuring morphological and surface features that are extremely small. A three dimensional view can be obtained by AFM.

4. Surface tension

5. Crystallization and melting behaviour: Differental Scanning Calorimetry (DSC) is used to study the melting behavior a drecrystallization of lipids. The more liquid lipid the less ordered the crystalline structure. X-ray diffraction technique is also used to know the status of lipids used.

6. Mobility and interactions : Mobility and interactions of solid lipid and liquid lipid is done by proton Nuclear Magnetic Resonance (NMR)

7. Molecular vibrations: Raman spectroscopy serves as tool in identification of molecular vibrations to detect high degree conformational order in NLC.
8. Molecular environment: Using the dye Nile red in fluorescent spectroscopy molecular environment or polarity of NLC is elucidated.

9. Drug loading efficiency/ encapsulation efficiency: This is determined by ultrafiltration, ultracentrifugation, gel filtration by sephadex, dialysis method. It is an important tool as it affects the release characteristics.

10. Drug release: The drug release behavior from NLCs is dependent upon the production temperature, emulsifier composition, and oil percentage incorporated in the lipid matrix. The drug amount in the outer shell of the nanoparticles and on the particulate surface is released in a burst manner, while the drug incorporated into the particulate core is released in a prolonged way. Dialysis method and utilization of franz cell are important methods to calculate drug release.

\section{APPLICATIONS: ${ }^{27,71,72 .}$}

In the arena of drug delivery systems, the nlc has occupied unique place spreading its wings in wide areas to mitigate number of diseases. By delivering the drug through various routes NLC is finding its way to treat diseases.

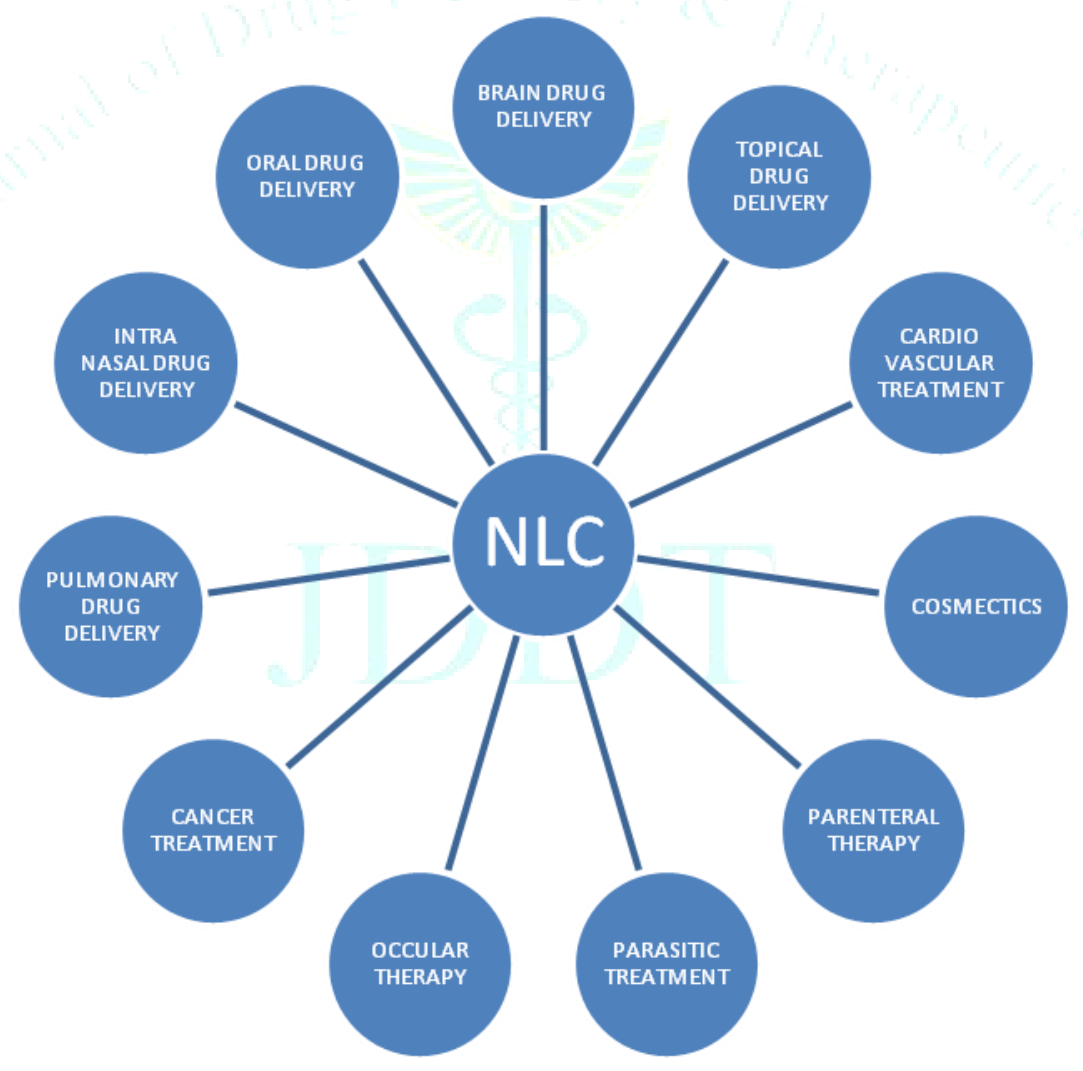

\section{REFERENCES:}

1. Uner M, "Preparation, characterization and physico-chemical properties of Solid Lipid Nanoparticles (SLN) and Nanostructured Lipid Carriers (NLC): Their benefits as colloidal drug carrier systems" Pharmazie, 2006; 61: 375-386.

2. Scheffel U, Rhodes BA, Natajaran TK, Wagner HN "Albumin microspheres for study of the reticuloendothelial system"J Nucl Med. 1970; 13:498-503.

3. Jumaa M, Muller BW "Lipid emulsions as a novel system to reduce the hemolytic activity of lytic agents: Mechanism of protective effect" Eur J Pharm Sci. 2000; 9: 285-90.
4. Parashar AK, D. Chadhar KV, Devaliya R, Shrivastav V, Jain UK, "A review on Solid Lipid Nanoparticles (SLN) for controlled and targeted delivery of medicinal agents Current Research in Pharmaceutical Sciences" 2011; 02: 37-47.

5. Manjunath K, Venkateswarlu V. "Pharmacokinetics, tissue distribution and bioavailability of clozapine solid lipid nanoparticles after intravenous and intraduodenal administration. J Control Release" 2005, 107:215-28.

6. Siekmann B, Westesen K. "Submicron-sized parenteral carrier systems based on solid lipids. Pharm Pharmacol Lett"1992, $1: 123-6$ 
7. Müller RH, Keck CM. "Challenges and solutions for the delivery of biotech drugs - a review of drug nanocrystal technology and lipid nanoparticles" J Biotech, 2004; 113:151-70.

8. Muller RH, Mäder K, Gohla S "Solid lipid nanoparticles (SLN) for controlled drug delivery-a review of the state of the art. Eur J Pharm Biopharm" 2000; 50:161-77

9. Diederichs JE, Muller RH "Liposomes in cosmetics and pharmaceuti-cal products. Pharm Indones" 1994; 56: 267-75.

10. Freitas C, Müller RH "Effect of light and temperature on zeta potential and physical stability in solid lipid nanoparticles (SLN) dispersions" Int J Pharm 1998; 168: 221-9.

11. Muller RH, Mäder K, Gohla S. "Solid lipid nanoparticles (SLN) for controlled drug delivery-a review of the state of the art" Eur J Pharm Biopharm 2000; 50: 161-77.

12. Muller RH, Mehnert W, Lucks JS, Schwarz C, Muhlen AZ, Weyhers $\mathrm{H}$ "Solid lipid nanoparticles (SLN)-alternative colloidal carrier systems for controlled drug delivery" Eur J Pharm Biopharm 1995; 41:62-9.

13. Cavalli R, Caputo O, Gasco MR "Solid lipospheres of doxorubicin and idarubicin" Int J Pharm 1993; 89: R9-R12.

14. Allen DD, Lockman PR, Oyewumi MO, Koziara JM, Roder KE, Mumper RJ "Brain uptake of thiamine-coated nanoparticles" Controlled Release 2003; 93: 271-82.

15. Dingler A "Feste lipid-nano patikelals colloidal works off trager system zur dermalen applikation, in PhD thesis" Berlin; 1998.

16. Fundaro A, Cavalli R, Bagoni A, Vighetto D, Zara GP, Gasco MR "Non-stealth and stealth solid lipid nanoparticles(sln) carrying doxorubicin: pharmacokinetic and tissue distribution after i. v. administration to rats" Pharm Res 2000; 42:337-43.

17. Chen D, Yang T, Liang W, Zhang Q "In vitro and in vivo study of two types of long circulating solid lipid nanoparticles containing paclitaxel" Chem Pharm Bull 2001; 49:1444-7.

18. Reddy JS, Venkateshwarlu V "Novel delivery systems for drug targeting to the brain" Drugs Future 2004; 29:63-83.

19. Wang JX, Sun X, Zhang ZR "Enhanced brain targeting by synthesis of 3',5'-dioctanoyl-5-fluoro-2'-deoxyuridine and incorporation into solid lipid nanoparticles" Eur J Pharm Biopharm 2002; 54:285-90

20. Siekmann B, Westesen K "Investigations on solid lipid nanoparticles prepared by precipitation in o/w emulsions" Eur J Pharm Biopharm 1996; 43:104-9.

21. Sarangi MK, Padhi S, "Solid Lipid Nanoparticles-A Review" Journal of Critical Reviews 2016; 3(3):5-12.

22. Yener MUG, "Importance of solid lipid nanoparticles (SLN) in various administration routes and future perspectives" International Journal of Nanomedicine 2007; 2(3):289-300.

23. Mukherjee S, Ray. S, Thakur R. S "Solid lipid nanoparticles: A modern formulation approach in drug delivery system" Indian Pharm Sci. 2009; 71(4):349-358.

24. Müller RH, Radtke M, Wissing SA "Nanostructured lipid matrices for improved microencapsulation of drugs" Int J Pharm 2002; 242:121-128.

25. Radtke M, Müller RH "Nanostructured Lipid Carriers: A novel generation of solid lipid drug drug Carriers" Pharmaceutical Technology Europe 1991; 17:1-4

26. Charcosset C, El-Harati A, Fessi H "Preparation of solid lipid nanoparticles using a membrane contactor" J Control Release , 2005; 108:112-120.

27. Kaur S, Nautyal U, Singh R, Singh S, Devi A. "Nanostructure Lipid Carrier (Nlc): The New Generation Of Lipid Nanoparticles" Asian Pacific Journal Of Health Sciences, 2015; 2(2):76-93.

28. Zhuang C Ning Li, Mi Wang, Xiao-Ning Zhang, Wei-San Pan, JunJie Peng, Yu-Sheng Pan, Xin Tang "Preparation and characterization of vinpocetine loaded nanostructured lipid carriers (NLC) for improved oral bioavailability" Int J Pharm. 2010; 394(1-2):179-185.

29. Tiwari R, Pathak K. "Nanostructured lipid carrier versus solid lipid nanoparticles of simvastatin: comparative analysis of characteristics, pharmacokinetics, and tissue uptake", Int J Pharm. 2011; 415(1-2):232-43.

30. Peddinti S "Nanostructured lipid carriers as a drug carrier" J Pharm Nanotechnol Nanostruc 2016; 4:68-74.

31. Araújo J, Gonzalez E , Egea MA , Garcia ML , Souto EB "Nanomedicinesfor ocular NSAIDs: safety on drug delivery" Nanomedicine. 2009; 5:394 - 401

32. Müller RH, Runge SA, Ravelli V. German Patent Application DE 19819273 A1; 1998
33. H. Bunjes, K.Westesen and M.H Koch, "Crystallization tendency and polymorphic transition in triglyceride nanoparticles" Int. J. Pharm, 1996; 129:159-173.

34. Patidar A, Thakur D, Kumar P and Verma J, “A review on Novel Lipid based Nanocarrier", International Jouranal of Pharmacy and Pharmacetical Science, 2010; 2(2):30-35.

35. Tamjidi F, Shahedi M, Varshosaz J et al. Nanostructured lipid carriers (NLC): a potential delivery system for bioactive food molecules.Innov. Food Sci. Emerg. Technol. 2013; 19:29-43.

36. Araújo J , Gonzalez E , Egea MA , Garcia ML ,Souto EB., Nanomedicinesfor ocular NSAIDs: safety on drug delivery Nanomedicine. 2009; 5:394 - 401.

37. Schafer-Korting M , Mehnert W , Korting HC., Lipid nanoparticles for improved topical application of drugs for skin diseases . Adv Drug Deliv Rev.2007; 59:427-443.

38. Radtke M, Muller RH "Nanostructured lipid carriers: the new generation of lipid drug carriers" New Drugs, 2001; 2: 48-52.

39. Jenning V, Thu..nemann AF, Gohla SH "Characterization of a novel solid lipid nanoparticle carrier system based on binary mixtures of liquid and solid lipids" Int J Pharm ,2000;199: 167177.

40. Müller R.H. et al., German patent application 2000; 199(45):203.2

41. Müller R.H. et al., extended patent on the basis of (6), PCT application PCT/EP00/04112 (2000). Drug release heading references.

42. Tamjidi F, Shahedi M, Varshosaz J, "Nanostructured lipid carriers (NLC): a potential delivery system for bioactive food molecules" Innov. Food Sci. Emerg.Technol; 2013; 19:29-43.

43. Ranpise NS, Korabu SS, Ghodake VN. 'Second generation lipid nanoparticles (NLC) as an oral drug carrier for delivery of lercanidipine hydrochloride". Colloids Surf. B Biointerfaces, 2014; 116:81-87.

44. Iqbal MA, Md S, Sahni JK, Baboota S, Dang S, Ali J "Nanostructured lipid carriers system: recent advances in drug delivery" J Drug Target. 2012; 20(10):813-830.

45. Fang CL, Al-Suwayeh SA, Fang JY "Nanostructured lipid carriers (NLCs) for drug delivery and targeting" Recent Pat Nanotechnol. 2013; 7(1):41-55.

46. Tran T.H, Ramasamy T, Truong D.H, Choi H.G, Yong C.S, Kim J.O "Preparation and characterization of fenofibrate-loaded nanostructured lipid carriers for oral bioavailability enhancement". AAPS Pharm.Sci. Tech, 2014; 15(6):1509-1515.

47. Chen CC, Tsai TH, Huang ZR, Fang JY, "Effects of lipophilic emulsifiers on the oral administration of lovastatin from nanostructured lipid carriers: physicochemical characterization and pharmacokinetics", Eur. J. Pharm. Biopharm, 2010; 74(3) 474-482.

48. Triplett MD, Rathman JF. Optimization of $\beta$-carotene loaded solid lipid nanoparticles preparation using a high shear homogenization technique. J. Nanopart. Res. 2008; 11(3):601614.

49. Hejri A, Khosravi A, Gharanjig K Hejazi M, "Optimisation of the formulation of beta-carotene loaded nanostructured lipid carriers prepared by solvent diffusion method" Food Chem. 2013; 141(1):117-123.

50. Tan SW, Billa N, Roberts CR, Burley JC, "Surfactant effects on the physical characteristics of amphotericin B-containing nanostructured lipid carriers" Colloids Surf. A Physicochem. Eng. Asp. 2010; 372(1-3):73-79.

51. Müller RH, Radtke M., Wissing SA, "Nanostructured lipid matrices for improved microencapsulation of drugs" Int. J. Pharm.2002; 242:121- 128.

52. Magdalene Radtke, Eliana B. Souto, Rainer H. Müller "Nanostructured Lipid Carriers: A Novel Generation of Solid Lipid Drug Carriers", Pharmaceutical Technology Europe, 2005; 17(4):45-50.

53. Dhruv K. Purohit, Tanaji D. Nandgude, Sushilkumar S. Poddar, "Nano-lipid Carriers for Topical Application: Current Scenario" Asian Journal of Pharmaceutics, 2016; (5):S3-S9.

54. Santos Maia C, Mehnert W, Schaller M, Korting HC, Gysler A Haberland A, Schafer Korting M "Drug targeting by solid lipid nanoparticles for dermal use” J Drug Target 2002; 10:489-95.

55. Pouton CW, Porter CJ "Formulation of lipid-based delivery systems for oral administration: Materials, methods and strategies" Adv Drug Deliv Rev 2008; 60:625-637. 
56. Miteva D, Joseph M Rutkowski, J Brandon Dixon, Witold Kilarski, Jacqueline D Shields, Melody A Swartz, "Transmural flow modulates cell and fluid transport functions of lymphatic endothelium" Circ Res, 2010; 106:920-931.

57. Iqbal J, Hussain M. "Intestinal Lipid Absorption" Am J Physiol Endocrinol Metab. 2009; 296(6):E1183-94.

58. Porter C, Natalie L Trevaskis, William N Charman "Lipid and lipid-based formulations: optimizing the oral delivery of lipophilic drugs" Nat Rev Drug Discov. 2007; 6:231.

59. Walker J, Anthony R. Odden, JeyaseelanS Zhang P , Gregory J. Bagby, Steve Nelson, and Kyle I. Happel "Ethanol exposure impairs LPS-induced pulmonary LIX expression: alveolar epithelial cell dysfunction as a consequence of acute intoxication" Alcohol Clin Exp Res. 2009; 33:357.

60. Vermeir M, Lachau-Durand S, Mannens G, Cuyckens F, Hoof BV, Raoof AR "Absorption, metabolism, and excretion of darunavir: a new protease inhibitor administered alone and with low dose ritonavir in healthy subjects" Drug Metab Dispos. 2009; 37:809.

61. Bhalekar M, Upadhaya P, Ashwini R Madgulkar, Sanjay J Kshirsagar, Akanksha Dube, Utkarsh S Bartakke "In-vivo bioavailability and lymphatic uptake evaluation for lipid nanoparticulates of darunavir", Drug Deliv; 2016; 23(7):2581.

62. Pandey P, Sharma A, and Gupta U "Blood brain barrier: an overview on strategies in drug delivery, realistic in-vitro modeling and in vivo live tracking", Tissue Barriers. 2016; (4):1, e1129476-1.
63. Radtke M, Müller RH "Stability study of creams containing cyclosporine SLN ${ }^{\mathrm{TM}}$. Drug Release from NLCs" Int Symp Control Rel Bioact Mater 2001; 28:472-473.

64. Banerjee D, Lao JY, Wang DZ, Huang JY, Ren ZF "Large-quantity free-standing ZnO nanowires". Appl Phys Lett, 2003; 83:2061.

65. Karuppasamy A, Subrahmanyam A, "Effect of electron bombardment on the properties of ZnO thin films" 2007, Mater Lett; 61(4-5):1256-1259.

66. Pearton SJ, Norton DP, Ip K, Heo YW, Steiner T “Recent progress in processing and properties of $\mathrm{ZnO}^{\prime}$ Prog Mater Sci, 2007; 50(3):293-340.

67. Ozgüra U, Alivov YI, Liu C, Tekeb A, Reshchikov MA "A comprehensive review of $\mathrm{ZnO}$ materials and devices" Journal of Applied Physics , 2005; 98:041301.

68. Lim SJ, Soon JK, Hyungjun K "High performance thin film transistor with low temperature atomic layer deposition nitrogen-doped ZnO" Appl Phys Lett 2007; 91:183517.

69. El Bekkaye Yousfi, Jacques F, Daniel L "Study of atomic layer epitaxy of zinc oxide by in-situ quartz crystal microgravimetry" Applied Surface Science, 2000; 153(4):223-234.

70. Seong KK, Cheol SH, Sang HK, Sun JY “Comparison between ZnO films grown by atomic layer deposition using $\mathrm{H} 2 \mathrm{O}$ or $\mathrm{O} 3$ as oxidant" Thin Solid Films, 2005; 478(1-2):103-108.

71. Natarajan. J, Karri VVSR and Anindita De "Nano structured Lipid carrier: A Promising Drug delivery system" Global Journal of Nano medicine, 2017; 1(5):1-5.

72. Chandana KV, Guta VN, Kanna S, "Nano structured Lipid carriers: The frontiers in drug delivery"Asian Journal of pharmaceutical and clinical research, 2019; 12(7):8-12. 